\title{
Las competencias lectoras del alumnado de educación primaria en riesgo de exclusión social: análisis de la producción científica
}

\author{
Magdalena Ramos-Navas-Parejo, M. Pilar Cáceres-Reche, José A. Marín-Marín y Carmen Rodríguez-Jiménez \\ Facultad de Ciencias de la Educación, Dpto. de Didáctica y Organización Escolar, Univ. de Granada, Campus \\ Universitario Cartuja s/n 180071 Granada, España. (Correo-e: magdalena@ugr.es; caceres@ugr.es; jmarin@ugr.es; \\ carmenrj@ugr.es)
}

Recibido Oct. 26, 2020; Aceptado Dic. 28, 2020; Versión final Feb. 3, 2021, Publicado Jun. 2021

\begin{abstract}
Resumen
El objetivo de este estudio es analizar la producción científica sobre las competencias lectoras del alumnado de educación primaria en los últimos 30 años. La adquisición de las competencias lectoras es fundamental en educación. Sin embargo, no todo el alumnado llega a adquirirlas. Se examinaron 824 referencias indexadas en Scopus entre los años 1990 y 2020. Los resultados muestran que al principio de los años noventa se publicaron entre $0(0 \%)$ documentos en 1991 y 7 documentos $(0,85 \%)$ en 1996. A partir del año 2005 , se produce una subida exponencial, que sigue una línea ascendente hasta llegar al máximo de 99 documentos $(12,01 \%)$ publicados en 2019 . Se concluye que se tiende a investigar cada vez más sobre las competencias lectoras del alumnado, especialmente a partir del año 2005, y que esta temática es objeto de interés de determinadas revistas, instituciones y autores.
\end{abstract}

\section{The reading skills of primary school students at risk of social exclusion: analysis of scientific production}

\begin{abstract}
The primary objective of this study is to examine the rate of scientific publications over the last 30 years on the topic of reading skills of primary school students. The acquisition of reading skills is fundamental in education. However, not all students acquire these skills. In total, 824 scientific articles were retrieved from Scopus that were published between the years 1990 and 2020. The results showed that, in the 90s, scientific publications on student reading skills ranged between zero $(0 \%)$ in 1991 and seven publications $(0.75 \%)$ in 1996 . From the year 2005, there was an exponential growth in publications until reaching a maximum of $99(12.1 \%)$ publications in 2019. It is concluded that there is a tendency to conduct more and more research on reading skills of primary school students, especially from the year 2005 and onwards, and that this is a subject of interest for specific journals, institutions, and authors.
\end{abstract}




\section{INTRODUCCIÓN}

La tendencia actual en investigación e innovación educativa va dirigida hacia la mejora de la práctica docente para dotarla de estrategias educativas que la lleven a atender a todo tipo de alumnado (González, 2016). Se tiende a una escuela comprensiva para garantizar una enseñanza de calidad a la totalidad del alumnado, que repercuta en el incremento del aprendizaje general y obtenga resultados óptimos en la consecución de los objetivos curriculares (Jiménez-García y Martínez-Ortega, 2017; Karlidag-Dennis et al., 2020). Sin embargo, en colegios situados en zonas desfavorecidas, estos objetivos están aún lejos de ser alcanzados. Aunque la escuela resulta ser el mayor agente socializador, las características familiares del alumnado de Educación Primaria en estos contextos suelen ser grandes obstáculos para la obtención de unos resultados educativos óptimos. Pese a que, como afirman Jiménez-García y Martínez-Ortega (2017) se han creado nuevos modelos de programas de atención a la diversidad y adaptaciones en las metodologías educativas, existen muchas carencias y urge encontrar soluciones efectivas a los problemas derivados de la diversidad cultural en las aulas, para alcanzar una educación equitativa de calidad.

La importancia de adquirir las competencias lectoescritoras ha sido referida por Triviño et al. (2013), Taghinezhad y Riasati (2020) y Hong et al. (2020), que las consideran uno de los procesos fundamentales en la formación escolar. Estos autores afirman que tales competencias ocupan un lugar destacado entre los aprendizajes escolares que están relacionados con ellas y la potencialidad del desarrollo de capacidades cognitivas y metacognitivas. Las competencias lectoescritoras son la base del resto de las competencias curriculares, tan importantes en educación, ya que la comprensión de enunciados de problemas matemáticos, por ejemplo, precisa de un nivel elevado de comprensión lectora. Alcanzar un buen nivel de competencia lectora, por tanto, puede ser un antídoto contra el fracaso escolar.

Los escolares pueden encontrar dificultades para conseguir una fluidez de lectura óptima debido a factores socioculturales, emocionales e intelectuales, o a dificultades específicas de aprendizaje, como la dislexia. Y su consecución es fundamental, ya que la fluidez es el primer paso para adquirir una lectura comprensiva, fundamental para el éxito académico, (Cain, 2016; Lai et al., 2014; Rodríguez, 2017). Por este motivo, es un objetivo prioritario de la educación obligatoria y una habilidad académica básica, junto con la escritura y el cálculo. La comprensión lectora, por tanto, se considera un proceso de orden superior y es el objetivo final de la lectura (Neumann et al., 2020), siendo el resultado de adquirir la capacidad de reconocer palabras escritas (decodificación visual) más la capacidad de comprensión del lenguaje oral (decodificación auditiva). Si alguno de estos dos aspectos es deficiente, la comprensión lectora también lo es. Esta es la conclusión a la que llegaron también Clarke et al. (2010) (figura1).

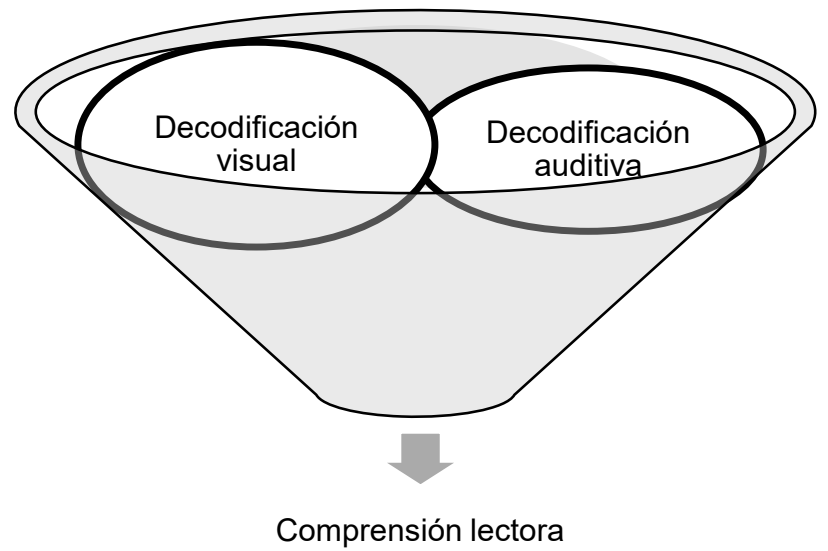

Fig. 1: Esquema de los componentes de la comprensión lectora según Clarke et al. (2010).

Retelsdorf y Köller, (2014) y Clarke et al. (2010) han estudiado los mecanismos de adquisición de la comprensión lectora y las dificultades para la obtención de la misma, con la finalidad de mejorarla. Sin embargo, las estrategias de mejora propuestas se reducen exclusivamente a tareas de evaluación (Calet et al., 2017). Según Ripoll y Aguado (2014) la forma más efectiva para mejorar la comprensión lectora es a través de la experiencia lectora (a la que se llega desde la motivación), el aumento del vocabulario y la mejora de la comprensión oral. Afirmación que comparten Jerrim, y Moss (2019) y Cain (2016) entre otros. Serna et al. (2017) insisten, también, en la importancia de la motivación lectora en niños y niñas de Educación Primaria que les lleve a la adquisición del hábito de la lectura de forma significativa; que es el que se realiza por placer y no por obligación. Proponen, junto con numerosos autores como Igwesi et al. (2012), Li et al. (2018), SeculGiust y Viñas (2015), fomentar la lectura y escritura desde las Tecnologías de la Información y la Comunicación (TIC), aprovechando la atracción de los niños y niñas del siglo XXI por ellas, y teniendo en 
cuenta que hay que seguir las tendencias sociales y que las actuaciones que se han realizado hasta ahora no han obtenido, en líneas generales, resultados satisfactorios (Prasanna-Kumara, 2019).

Valdés (2013) defiende que si un menor en riesgo de exclusión social lograra adquirir el hábito lector evitaría no sólo el fracaso escolar, con la mejora de las habilidades lectoescritoras, sino que ello le permitiría seguir dentro del sistema social. Este autor considera que el alumnado que más ayuda necesita para adquirir el hábito lector es el perteneciente a una clase social desfavorecida. Y reitera que es justo en estos casos cuando más importante resulta esta adquisición que le abriría las puertas al sistema educativo, ayudando a la equidad educativa que nuestro sistema educativo ansía, y a la integración social. Fue a principios de 1990 cuando los documentalistas comenzaron a interesarse por realizar análisis bibliométricos (Miranda, 1990). A través de estos métodos estadísticos se pueden obtener parámetros con los cuales conocer el proceso de la comunicación escrita, así como la naturaleza y el curso del desarrollo de una disciplina. Autores como Rodríguez y Gallego (2019) también comparten la idea de que los análisis bibliométricos proporcionan una información relevante sobre el estado actual de un área de conocimiento, la productividad de las distintas universidades y la evolución de las revistas en ese mismo ámbito, entre otras cuestiones de interés.

Resultan, por tanto, que son fundamentales aquellos estudios de investigación en este ámbito que lleven a la innovación educativa, para realizar intervenciones bien diseñadas y coordinadas, en el ámbito de la motivación lectora en las escuelas, especialmente de contextos desfavorecidos, y así conseguir la mejora de una educación que incluya a todos y evite la exclusión social. En ello deberían implicarse y formarse también maestros, bibliotecarios y familiares para evitar contradicciones (Álvarez y Pascual 2013). Teniendo en cuenta estas consideraciones y su importancia en el ámbito educativo y social, en este trabajo se ha realizado un estudio bibliométrico con el objetivo de analizar la producción científica sobre los ámbitos de la lectura y la educación inclusiva, existente en una de las bases de datos más relevantes (Scopus), en los últimos 30 años.

Con este trabajo se pretende responder a las siguientes cuestiones: ¿cuál ha sido la evolución de las publicaciones sobre esta temática a través del tiempo en la base de datos Scopus?, ¿existen autores y revistas especializadas en este ámbito de la educación?, ¿qué disciplinas están relacionadas con la temática?, ¿cuál es la tipología de documentos publicados?, ¿qué países e instituciones están más involucrados con esta área educativa?, ¿qué revistas destacan en la indexación estos artículos que tratan sobre la comprensión lectora atendiendo a la inclusión educativa?, ¿cuáles son los idiomas de publicación más relevantes?, ¿cuáles son los artículos más citados y los autores con mayor producción?', ¿qué relación existe entre las distintas palabras clave de los artículos analizados?

\section{METODOLOGÍA}

La metodología que se ha utilizado para la realización de este análisis bibliométrico está basada en la propia de este tipo de estudios, en los cuales se cuantifican los documentos sobre el ámbito concreto a investigar publicados en diversas bases de datos. En este caso se ha analizado la producción sobre la adquisición de las competencias lectoras en situaciones desfavorables, de la prestigiosa base de datos Scopus. Se trata de un estudio de tipo descriptivo, de carácter transversal-retrospectivo.

Tras seleccionar los descriptores más apropiados para definir el objeto de estudio e indexarlos en el tesauro de Eric, para verificar que coinciden con las palabras clave más utilizadas en el lenguaje científico, se realizaron las ecuaciones de búsqueda. Los descriptores utilizados fueron: Reading comprehension (Comprensión lectora), Reading habits (Hábitos de lectura), Academic Achievement (Logro académico), Educational disavantage (Desventaja educativa), Social integration (Integración social), Educational needs (Necesidades educativas), Special need students (Estudiantes con necesidades especiales). Se realizaron diferentes combinaciones en el buscador de Scopus, buscando un número representativo de documentos, que permita la ejecución de este trabajo y que defina el objetivo del estudio. En este sentido, se seleccionaron las palabras clave Reading comprehension y Educational needs. Estas se conectaron utilizando el operador booleano and, dentro de la opción all fields.

La obtención de los datos y su análisis han tenido lugar durante el mes de agosto de 2020. De esta forma, se han analizado distintos indicadores bibliométricos, atendiendo a los antecedentes de otros similares (Aznar et al., 2018; Campos-Soto et al., 2020): 1) Indicadores de producción: como la productividad diacrónica y personal, las cuales han cotejado las leyes de Price y de Lotka; 2) Indicadores de dispersión: con los que se ha verificado la ley de Bradford y 3) Indicadores de impacto: el área de publicación, tipología de los documentos, país de publicación, afiliación de los autores, revistas de indexación, idioma de publicación, artículos más citados y autores con más producción.

Además, se han analizado las conexiones que existen entre las palabras claves de los artículos estudiados en este trabajo, los cuales versan sobre la lectura y su relación con las necesidades educativas. Se ha podido confeccionar un mapa de redes con la utilización del software VOSviewer. A través de la exportación desde 
Scopus de todas las palabras clave en inglés, esta aplicación puede relacionar todas ellas y representarlas en una figura gráfica que ayuda de forma muy visual a comprender estas conexiones entre descriptores, lo que aporta información sobre los campos de la investigación en los que están los artículos insertados, entre otros datos de interés. Para la confección de la muestra final $(n=824)$ se tuvieron en consideración los diferentes criterios de inclusión, reflejados en la tabla 1, los cuales se han decidido en función de los objetivos y preguntas de investigación. La muestra es equivalente a la población, puesto que han sido analizados todos los documentos.

Tabla 1: Criterios de inclusión en función de las variables

\begin{tabular}{|l|l|}
\hline \multicolumn{1}{|c|}{ Variables } & \multicolumn{1}{c|}{ Criterios de inclusión } \\
\hline $\begin{array}{l}\text {-Año de } \\
\text { publicación }\end{array}$ & $\begin{array}{l}\text {-Los años comprendidos entre 1990 y 2020, (aunque el año 2020 está } \\
\text { incompleto, queda incluido hasta el mes de agosto). }\end{array}$ \\
\hline $\begin{array}{l}\text {-Tipología de los } \\
\text { documentos }\end{array}$ & $\begin{array}{l}\text {-Para analizar los indicadores de dispersión, la productividad personal y } \\
\text { realizar el mapa de redes, solo se tuvieron en cuenta los artículos } \\
\text { científicos. Para el resto del estudio se han utilizado todos los documentos }\end{array}$ \\
\hline $\begin{array}{l}\text {-Áreas de } \\
\text { publicación }\end{array}$ & -Se han contemplado a partir de 10 documentos \\
\hline -País & $\begin{array}{l}\text {-Se han seleccionado los que tienen publicados a partir de 14 documentos } \\
\text { (desde el 1,70\%) }\end{array}$ \\
\hline -Afiliación & -Las que tienen a partir de 9 documentos (desde el 1\%) \\
\hline -Revistas & -Las que tienen a partir de 8 documentos publicados \\
\hline -Idiomas & -Se han contemplado todos \\
\hline -Referencias & -Se han analizado las 5 más citadas \\
\hline -Autores & -Los que han publicado a partir de 5 documentos \\
\hline -Mapa bibliométrico & $\begin{array}{l}\text {-Se ha realizado con las palabras clave de los artículos indexados en } \\
\text { Scopus }\end{array}$ \\
\hline
\end{tabular}

Los datos se han extraído de la base de datos Scopus, los cuales se han organizado utilizando las hojas de cálculo de Excel y se han analizado a través del programa estadístico SPSS 25 , con el que se han obtenido porcentajes de aparición y regresión lineal de variables, representados en gráficos de dispersión con la finalidad de comparar valores y hallar las líneas de tendencia. Se han utilizado estadísticos como: porcentajes, coeficiente de correlación de Pearson y coeficiente de regresión.

\section{RESULTADOS}

Para poder implementar este estudio con una cantidad de documentos suficiente para llevarlo a cabo y lo más próximo a la temática que se pretende analizar, se escogió la combinación Reading comprehension y Educational needs. De esta forma, se acota esta competencia lectora a su relación con las dificultades educativas en términos generales. Los resultados de la búsqueda se obtuvieron de la combinación de estos descriptores en la base de datos Scopus, a través de la selección de la opción "todos los campos" y el conector "y" para aumentar el rigor de la búsqueda. Lo que obtuvo como resultado un total de 831 referencias, que al aplicar los criterios de inclusión y exclusión se vio reducido a 824 documentos.

\section{Indicadores de producción}

Dentro de los indicadores de producción se han analizado la producción diacrónica y la productividad personal de las referencias halladas en Scopus sobre la temática estudiada.

\section{Producción diacrónica}

Tras analizar las 824 referencias indexadas en la base de datos Scopus, que se obtienen con la combinación de los descriptores seleccionados, acotando la búsqueda a los años comprendidos entre 1990 y 2020 y utilizando como variable "el año de publicación", podemos observar el aumento en los últimos 30 años de la producción científica que trata la comprensión lectora desde las necesidades educativas. Observando estos resultados se comprueba como al principio de los años noventa se publican entre $0(0 \%)$ documentos en 1991 y 7 documentos $(0,85 \%)$ en 1996, subiendo la producción progresivamente con el paso de los años (Figura 2). A partir del año 2005 , se produce una subida exponencial, que sigue una línea ascendente hasta llegar al máximo de 99 documentos (12,01\%) publicados en 2019. Teniendo en cuenta que el año 2020 se encuentra en curso y que hasta el mes de agosto ya cuenta con 84 documentos $(10,19 \%)$, resulta sencillo prever que al finalizar seguirá la tendencia ascendente que marca la gráfica. 
Si se atiende a la ley de Price, se puede comprobar el cumplimiento de la premisa que afirma que la producción científica se duplica cada 10-15 años (Price, 1986). En este caso, la proporción es incluso mayor; de 1 documento publicado en $1990(0,12 \%)$ a los diez años se pasa a $7(0,85 \%)$, que corresponde al año $2000,41(4,98 \%)$ en 2010 y $84(10,19 \%)$ en el año inacabado 2020 . Al sumar las referencias halladas en la primera década analizada, se obtienen 29 en total (3,52\%), en la segunda ascienden a $110(13,35 \%)$ y en la tercera década se han publicado 665 documentos que suponen el $80,70 \%$ del total de las referencias estudiadas. Incluso estas últimas cifras se verán aumentas al finalizar el año 2020. Estos datos obtenidos dan clara cuenta del incremento exponencial de las publicaciones que se han realizado en los últimos 30 años en Scopus sobre el tema que se analiza en este estudio bibliométrico, confirmando la ley de Price.

El descenso de este año en curso se explica por el hecho de que aún no ha terminado, por tanto, solo consta de 7 meses completos, a diferencia del resto de los que se analizan los 12 meses íntegros.



Fig. 2: Productividad diacrónica desde 1990 hasta el año 2020

\section{Productividad personal}

Con respecto a la productividad personal, se comprueba que se cumple la ley de Lotka, que afirma que el grueso de los artículos publicados sobre un determinado tema coincide con un número muy reducido de autores que se encuentran especializados en esa área de conocimiento. Como se observa en la figura 3, la correlación entre el menor número de autores y el mayor número de artículos de Scopus es positiva. Así se aprecia en un extremo, que 2 autores han publicado 11 artículos y en el extremo opuesto más de 230 autores han publicado uno. Al examinar la relación lineal entre las variables, se obtiene un coeficiente de correlación de Pearson $r=-0,6705$, lo que indica que existe una dependencia media-alta entre las variables y que poseen una correlación negativa, ya que se afectan de forma inversamente proporcional. Con respecto al coeficiente de determinación o de correlación múltiple el resultado obtenido es $\mathrm{R}^{2}=0.9219$, lo que indica que tiene muy buen ajuste.

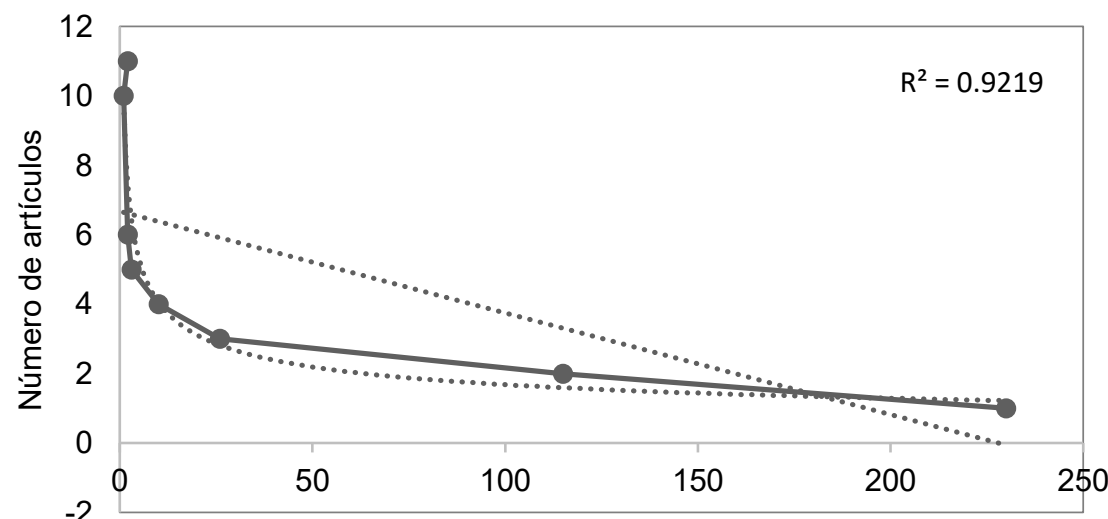

Número de autores

Fig. 3: Correlación entre número de autores y el número de artículos Scopus 


\section{Indicadores de dispersión}

El análisis de los indicadores de dispersión revela que se cumple la premisa que define la ley de Bradford o ley de dispersión de la literatura científica de Bradford, la cual analiza la relación cuantitativa entre las revistas científicas y los artículos indexados en las mismas sobre una temática concreta, afirmando que un pequeño número de revistas especializadas contienen el mayor número de artículos. De forma que si se organizan en un diagrama de dispersión donde existe un núcleo con un número de artículos similar al resto de las zonas, el número de fuentes donde se encuentran indexados en las áreas de mayor concentración es menor. Esta cifra va aumentando conforme se entra en bandas de mayor dispersión, más alejadas del núcleo (Miranda, 1990). Para determinar estas zonas se han seleccionado en Scopus, dentro de tipo de documento, los artículos de revistas exclusivamente. Después, se ha filtrado por tipo de fuente, obteniendo el nombre de cada revista y el número de artículos que contiene cada una. El número de documentos se ha dividido entre 5 para delimitar las zonas. Y por último se ha averiguado el número de revistas que contiene cada zona.

El total es de 322 revistas y 601 artículos (Fig. 4), distribuidos en 5 zonas con una media de 120 artículos, aproximadamente, en cada una, donde se puede observar que el núcleo lo conforman solamente 12 revistas, conteniendo una cantidad similar de documentos que el resto de áreas que tienen 98 o incluso 128 revistas, en las zonas más alejadas del núcleo. El análisis de regresión lineal muestra una media-alta correlación negativa existente entre el número de revistas y los artículos acumulados (Fig. 5). Tiene un coeficiente de correlación de Pearson $r=-0,6863$. $Y$ un coeficiente de determinación o correlación múltiple $R^{2}=0,9275$, lo que indica un ajuste muy bueno.

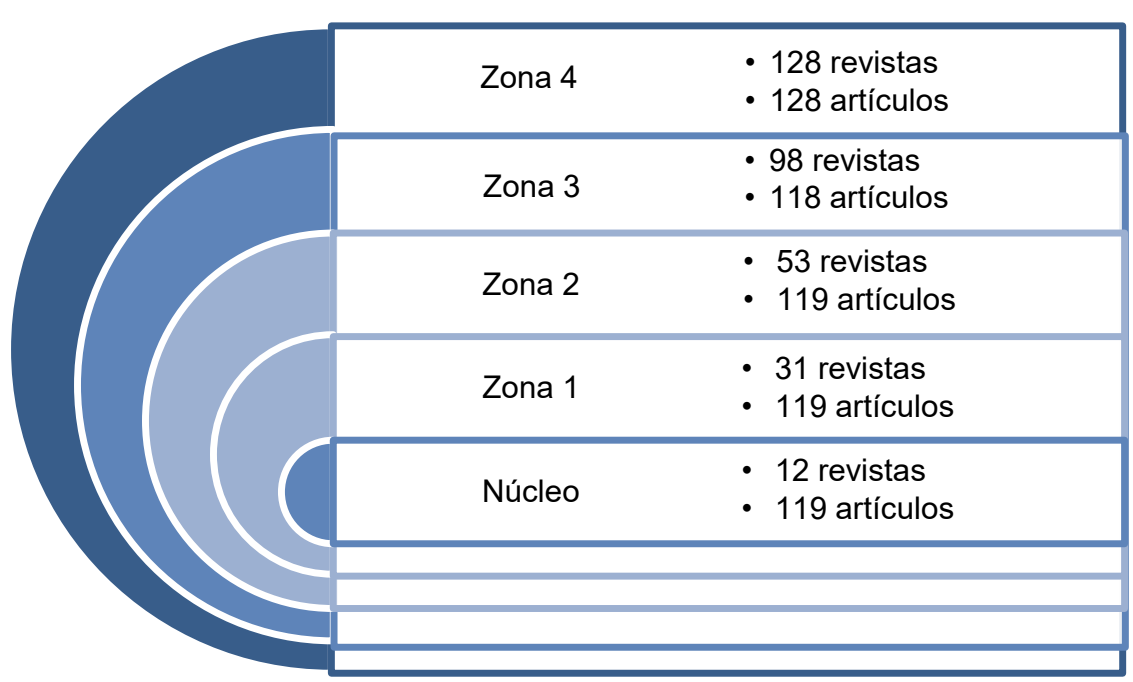

Fig. 4: Área de dispersión de Bradford de las revistas científicas que tratan la comprensión lectora atendiendo a las necesidades educativas.

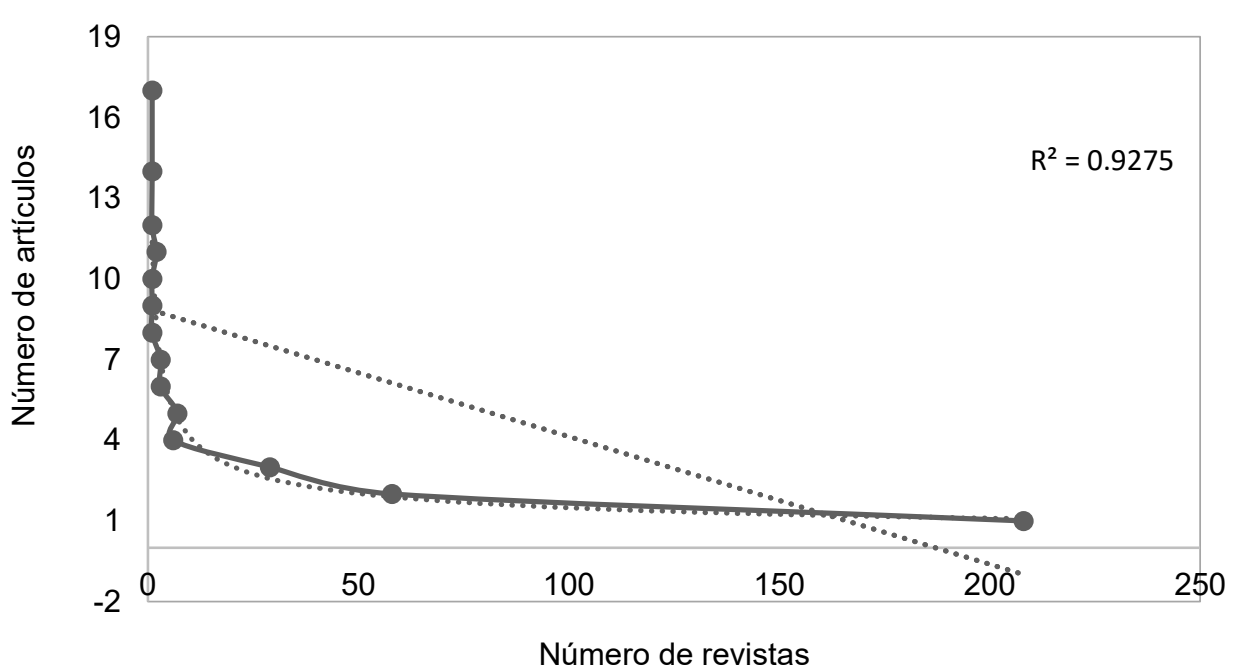

Fig. 5: Análisis de regresión lineal entre el número de revistas y el número de artículos 


\section{Indicadores de impacto}

Con la intención de conocer la influencia que tienen los estudios que relacionan las competencias lectoras con el alumnado en riesgo de exclusión social, se han analizado diferentes indicadores de impacto como son: el área de publicación, tipo de documentos, país de publicación, afiliación, revistas de mayor impacto, idioma de publicación y artículos más citados.

\section{Área de publicación}

Las distintas referencias se encuentran indexadas en diferentes áreas de publicación, incluso algunas se pueden encontrar en varias categorías. De forma global se puede observar que la mayoría de la producción científica sobre este tema se encuentra en el área de las Ciencias Sociales $(70,02 \%)$ seguido del área de la Psicología (42,35\%) y de la Medicina (12,38\%). Datos que se representan en la tabla 2 , que recoge las áreas de publicación que contienen a partir de 10 documentos de las 22 áreas en las que se encuentran repartidas las 824 referencias analizadas. Cabe destacar que los porcentajes se han realizado sobre el total de documentos en cada área de publicación, pero puesto que algunos documentos pueden encontrase dentro de varias categorías la suma de los porcentajes no coincide con el $100 \%$ de los artículos.

Tabla 2: Número de documentos en función del área de publicación en la base de datos Scopus

\begin{tabular}{|l|c|c|}
\hline \multicolumn{1}{|c|}{ Área de Publicación } & $N^{\circ}$ de documentos & Porcentaje total \\
\hline Ciencias sociales & 577 & $70,02 \%$ \\
\hline Psicología & 349 & $42,35 \%$ \\
\hline Medicina & 102 & $12,38 \%$ \\
\hline Artes y Humanidades & 88 & $10,68 \%$ \\
\hline Profesiones de la Salud & 78 & $9,47 \%$ \\
\hline Ciencias de la Computación & 48 & $5,82 \%$ \\
\hline Neurociencia & 28 & $3,40 \%$ \\
\hline Ingeniería & 16 & $1,94 \%$ \\
\hline Matemáticas & 10 & $1,21 \%$ \\
\hline
\end{tabular}

Tipo de documento

Al analizar los distintos tipos de documentos (Tabla 3) se comprueba que la mayoría son artículos científicos (72,94\%), seguido, a mucha distancia, de libros $(9,10 \%)$, revisiones, capítulos de libro, documentos de sesión, notas y editoriales. Se puede afirmar, por tanto, que el formato preferente para publicar estudios de investigación sobre la comprensión lectora asociada a las necesidades educativas es el artículo de divulgación científica.

Tabla 3: Número de documentos en función de la tipología de los documentos hallados en Scopus

\begin{tabular}{|l|c|c|}
\hline \multicolumn{1}{|c|}{ Tipo documento } & $N^{\circ}$ de documentos & Porcentaje \\
\hline Artículo & 601 & $72,94 \%$ \\
\hline Libro & 75 & $9,10 \%$ \\
\hline Revisión & 60 & $7,28 \%$ \\
\hline Capítulo de libro & 59 & $7,16 \%$ \\
\hline Documento de Sesión & 21 & $2,55 \%$ \\
\hline Nota & 4 & $0,48 \%$ \\
\hline Editorial & 2 & $0,24 \%$ \\
\hline Indefinido & 2 & $0,24 \%$ \\
\hline
\end{tabular}

\section{País de publicación}

Los países con mayor producción científica sobre la comprensión lectora atendiendo a las necesidades educativas son Estados Unidos (28,28\%) y Reino Unido (22,69\%), como se puede apreciar en la tabla 4, en la que se representan los países que tienen a partir de 14 documentos publicados sobre la temática que compete a este trabajo en los últimos 30 años. De esta forma se puede observar el panorama internacional, localizando geográficamente las instituciones que más investigan sobre esta área. Se han extraído 14 países que contienen 711 documentos de los 824 analizados, lo que equivale a un $86,29 \%$ del total. 
Tabla 4: Número de documentos indexados en función del país de publicación en la base de datos Scopus

\begin{tabular}{|l|c|c|}
\hline \multicolumn{1}{|c|}{ Países } & $N^{\circ}$ de documentos & Porcentaje \\
\hline Estados Unidos & 233 & $28,28 \%$ \\
\hline Reino Unido & 187 & $22,69 \%$ \\
\hline Australia & 51 & $6,19 \%$ \\
\hline Canadá & 39 & $4,73 \%$ \\
\hline Alemania & 35 & $4,25 \%$ \\
\hline España & 27 & $3,28 \%$ \\
\hline Suecia & 23 & $2,79 \%$ \\
\hline Finlandia & 21 & $2,55 \%$ \\
\hline Países Bajos & 20 & $2,43 \%$ \\
\hline Nueva Zelanda & 19 & $2,31 \%$ \\
\hline Brasil & 14 & $1,70 \%$ \\
\hline Grecia & 14 & $1,70 \%$ \\
\hline Irlanda & 14 & $1,70 \%$ \\
\hline Italia & 14 & $1,70 \%$ \\
\hline
\end{tabular}

\section{Afiliación}

La siguiente variable a analizar es la institución a las que están afiliados los autores de los documentos encontrados en la base de datos Scopus. Como cabe esperar, puesto que el $50,97 \%$ de todos los documentos publicados se encuentran repartidos entre Estados Unidos y el Reino Unido, las principales instituciones pertenecen a estos dos países. En la tabla 5 se han recogido las afiliaciones de los autores que contienen a partir de 9 documentos (1,09\%) estableciendo este corte a partir del 1\% del total. El número de referencias repartidas entre las 12 instituciones con más de 9 afiliaciones son 148, un 17,96\% del total (824).

El mayor número de citas $(2153)$ y de impacto $(113,32)$ lo recoge la Universidad de Durham en el Reino Unido, seguido de la Universidad de Bristol, perteneciente al mismo país, con 987 citas y un impacto de 98,70. Las principales universidades a las que están afiliados los autores, que se recogen en la tabla 5, pertenecen a Estados Unidos y el Reino Unido, excepto la Universidad de Jyvaskyla, que se encuentra en Finlandia, país destacado por sus altos resultados en las pruebas PISA y la Universidad de Canterbury, perteneciente a Nueva Zelanda.

Tabla 5: Número de documentos en función de la afiliación encontrados en la base de datos de Scopus

\begin{tabular}{|l|c|c|c|c|}
\hline \multicolumn{1}{|c|}{ Afiliación } & $N^{\circ}$ de documentos & $\%$ & Citas & Índice de impacto \\
\hline UCL Institute of Education & 25 & 3,03 & 820 & 32,80 \\
\hline Durham University & 19 & 2,31 & 2153 & 113,32 \\
\hline University of Texas at Austin & 14 & 1,70 & 249 & 17,79 \\
\hline University College London & 13 & 1,58 & 164 & 12,61 \\
\hline Vanderbilt University & 10 & 1,21 & 265 & 26,50 \\
\hline University of Canterbury & 10 & 1,21 & 138 & 13,80 \\
\hline University of Bristol & 10 & 1,21 & 987 & 98,70 \\
\hline University of Oxford & 10 & 1,21 & 167 & 16,70 \\
\hline University of Jyvaskyla & 10 & 1,21 & 251 & 25,10 \\
\hline Florida State University & 9 & 1,09 & 194 & 21,55 \\
\hline University of York & 9 & 1,09 & 220 & 24,44 \\
\hline University of London & 9 & 1,09 & 228 & 25,33 \\
\hline
\end{tabular}

\section{Revistas}

Las revistas que recogen más de un $0,9 \%$ de los documentos analizados son las 10 que se pueden observar en la tabla 6, que contienen 112 referencias suponiendo el 13,59\% del total. Se puede apreciar que la fuente que contiene mayor número de citas $(263)$ es la revista Dyslexia. Sin embargo, el mayor impacto $(25,75)$ recae en la revista Educational and Child Psychology. La revista que recoge mayor número de documentos es Journal of Research in Special Educational Needs en la cual se encuentran 17 de los 824 documentos estudiados, suponiendo un $2,06 \%$ del total. 
Tabla 6: Número de documentos en las principales revistas de Scopus y WoS

\begin{tabular}{|l|c|c|c|c|}
\hline \multicolumn{1}{|c|}{ Revistas } & $N^{\circ}$ de documentos & $\%$ & $N^{\circ}$ citas & Índice de impacto \\
\hline Journal of Research in Special Educational Needs & 17 & 2,06 & 177 & 10,41 \\
\hline Dyslexia & 14 & 1,70 & 263 & 18,79 \\
\hline Frontiers in Psychology & 14 & 1,70 & 50 & 3,57 \\
\hline Research in Developmental Disabilities & 12 & 1,46 & 119 & 9,92 \\
\hline Journal of Learning Disabilities & 11 & 1,33 & 261 & 23,73 \\
\hline Reading and Writing & 11 & 1,33 & 227 & 20,64 \\
\hline Learning and Individual Differences & 9 & 1,09 & 132 & 14,67 \\
\hline Educational and Child Psychology & 8 & 0,97 & 206 & 25,75 \\
\hline Educational Psychology in Practice & 8 & 0,97 & 82 & 10,25 \\
\hline International Journal of Inclusive Education & 8 & 0,97 & 56 & 7,00 \\
\hline
\end{tabular}

\section{Idioma de publicación}

Otra variable que se ha analizado en esta investigación bibliométrica es la lengua en la que se han escrito y publicado los distintos documentos (Tabla 7). El inglés es el idioma predominante abarcando un 96,12\% del total de los documentos analizados. No sorprende si se tienen en cuenta los principales países de procedencia de los documentos (Estados Unidos y Reino Unido) y que el inglés es el idioma más utilizado en el ámbito de la investigación. Le sigue a mucha distancia el español (1,33\%) y el alemán $(0,97 \%)$.

Tabla 7: Número de documentos en función del idioma de publicación

\begin{tabular}{|l|c|c|}
\hline Idioma & $N^{\circ}$ de documentos & $\%$ \\
\hline Inglés & 792 & 96,12 \\
\hline Español & 11 & 1,33 \\
\hline Alemán & 8 & 0,97 \\
\hline Portugués & 6 & 0,73 \\
\hline Francés & 3 & 0,36 \\
\hline Italiano & 3 & 0,36 \\
\hline Húngaro & 2 & 0,24 \\
\hline Turco & 2 & 0,24 \\
\hline Africaans & 1 & 0,12 \\
\hline Ruso & 1 & 0,12 \\
\hline Serbio & 1 & 0,12 \\
\hline Esloveno & 1 & 0,12 \\
\hline
\end{tabular}

\section{Referencias más citadas}

Se han analizado los documentos más citados en las dos bases de datos. En la tabla 8 se pueden apreciar las 5 referencias con mayor número de citas de Scopus, los cuales se publicaron entre los años 2000 y el 2008.

Tabla 8: Documentos más citados de Scopus

\begin{tabular}{|l|c|l|l|c|}
\hline \multicolumn{1}{|c|}{ Autores } & Año & \multicolumn{1}{c|}{ Titulo } & \multicolumn{1}{c|}{$\begin{array}{c}N^{\circ} \text { decalización } \\
\text { citas }\end{array}$} \\
\hline Hattie & $(2008)$ & $\begin{array}{l}\text { Visible Learning: A Synthesis of Over 800 } \\
\text { Meta-Analyses Relating to Achievement }\end{array}$ & $\begin{array}{l}\text { Libro: University of } \\
\text { Auckland, New Zealand }\end{array}$ & 3292 \\
\hline $\begin{array}{l}\text { Gathercole, } \\
\text { Pickering, Knight y } \\
\text { Stegmann }\end{array}$ & $(2004)$ & $\begin{array}{l}\text { Working memory skills and educational } \\
\text { attainment: Evidence from national curriculum } \\
\text { assessments at 7 and 14 years of age }\end{array}$ & $\begin{array}{l}\text { Appliced Cognitive } \\
\text { Psychology }\end{array}$ & 461 \\
\hline $\begin{array}{l}\text { Gathercole, Alloway, } \\
\text { Willis y Adams }\end{array}$ & $(2006)$ & $\begin{array}{l}\text { Working memory in children with reading } \\
\text { disabilities }\end{array}$ & $\begin{array}{l}\text { Journal of Experimental } \\
\text { Child Psychology }\end{array}$ & 426 \\
\hline Murphy y Alexander & $(2000)$ & $\begin{array}{l}\text { A motivated exploration of motivation } \\
\text { terminology }\end{array}$ & $\begin{array}{l}\text { Contemporary } \\
\text { Educational Psychology }\end{array}$ & 399 \\
\hline $\begin{array}{l}\text { Zimmerman } \\
\text { y Christakis }\end{array}$ & $\mathbf{2 0 0 5 )}$ & $\begin{array}{l}\text { Children's television viewing and cognitive } \\
\text { outcomes: A longitudinal analysis of national } \\
\text { data }\end{array}$ & $\begin{array}{l}\text { Archives of Pediatrics } \\
\text { and Adolescent } \\
\text { Medicine }\end{array}$ & 258 \\
\hline
\end{tabular}




\section{Autores con mayor producción}

Los autores que han escrito más documentos sobre la comprensión lectora asociada a las necesidades educativas indexados en Scopus, se encuentran representados en la tabla 9, donde se han recogido los autores con más de 5 investigaciones sobre la temática. Como se puede comprobar Alloway y Gathercole son los autores que más han producido, con 11 documentos $(1,33 \%)$, seguido de Everatt con $10(1,21 \%)$. Gathercole es el autor más citado, con 1.562 citas y el que tiene mayor impacto (142). También se puede observar que es autor de dos de los cinco artículos más citados en Scopus sobre esta temática estudiada. Uno de estos artículos lo comparte con Alloway.

Tabla 9: Autores con mayor número de documentos

\begin{tabular}{|l|c|c|c|c|}
\hline Autores & $N^{\circ}$ documentos & $\%$ & Citas & Índice de impacto \\
\hline Gathercole & 11 & 1,33 & 1562 & 142,00 \\
\hline Alloway & 11 & 1,33 & 1050 & 95,45 \\
\hline Everatt & 10 & 1,21 & 274 & 27,40 \\
\hline Dockrell & 6 & 0,73 & 330 & 55,00 \\
\hline Elbeheri. & 6 & 0,73 & 67 & 11,17 \\
\hline Lindsay & 5 & 0,61 & 226 & 45,20 \\
\hline Mahfoudhi & 5 & 0,61 & 51 & 10,20 \\
\hline Woolley & 5 & 0,78 & 13 & 2,60 \\
\hline
\end{tabular}

\section{Mapa bibliométrico}

La figura 6 representa el mapa de redes de los principales descriptores utilizados por los documentos analizados y las relaciones entre ellos. Los colores distinguen cuatro temáticas diferentes en las que se engloban las palabras clave. Se puede observar que los conceptos poseen diferentes tamaños, los cuales representan la frecuencia de aparición y las conexiones con otros descriptores. En el clústers de color azul los conceptos de mayor tamaño son: human (humano), male (masculino) y adolescent (adolescente). Estos descriptores, como se puede comprobar, distan de la temática estudiada. En el clúster de color amarillo los descriptores no son muy relevantes y hacen mención a las áreas de las disciplinas y capacidades psicológicas. El grupo de color verde está muy relacionado con la educación y las necesidades educativas e inclusivas conectadas con la lingüística, destacando conceptos como: education (educación), learning (aprendizaje), teaching (enseñanza) y literacy (alfabetismo). Por su parte el clúster de color rojo hace referencia a diferentes aspectos de la psicología relacionada con el aprendizaje infantil, pudiendo observar que los conceptos más sobresalientes son: child (niño), children (niños), dyslexia (dislexia), working memory (memoria de trabajo), short term memory (memoria a corto plazo) y phonetics (fonética).

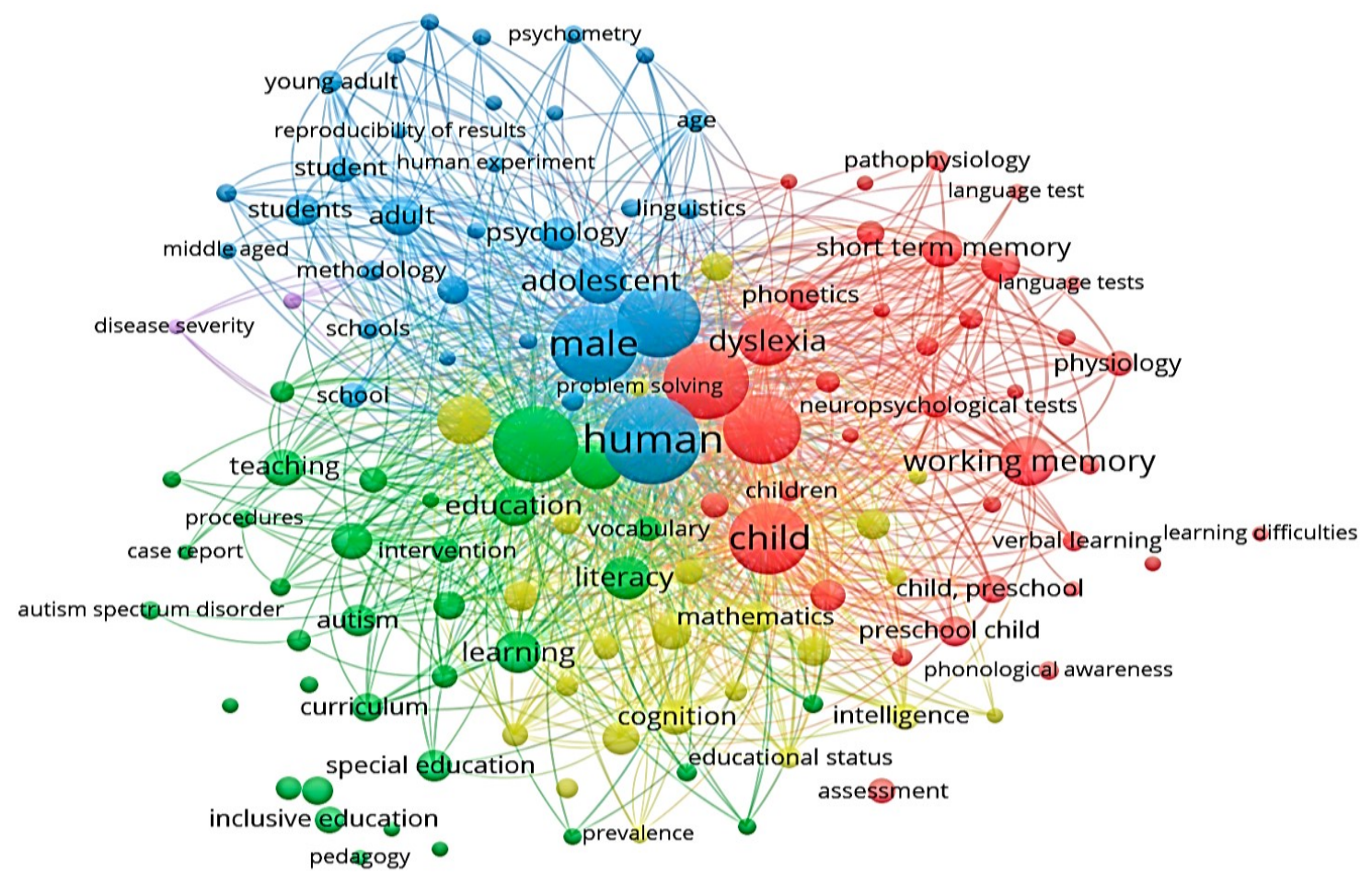

Fig.6: Mapa de redes entre las palabras clave de los artículos publicados sobre la comprensión lectora atendiendo a las necesidades educativas 
El mapa (Fig.6) se ha obtenido a través del programa Vosviewer. Este software genera un mapa de forma visual de las palabras clave de las distintas aportaciones científicas automáticamente, Resaltando las que más veces aparecen y su relación con los estudios a los que están conectadas. La nube de palabras y el mapa de redes son gráficos parecidos. La diferencia reside en que el primero no establece interrelaciones entre términos, solamente indica el número de veces que aparecen.

\section{CONCLUSIONES}

Tras estudiar los resultados derivados de esta investigación, su discusión, la constatación con los resultados de otros autores y el detallado análisis presentado, se extraen las siguientes conclusiones principales: 1) es muy reciente la producción científica sobre las dificultades de adquisición de las competencias lectoras en situaciones de desventaja educativa, ya que aun siendo un problema educativo que ha existido siempre, es desde hace unos 15 años cuando comienza a haber un volumen de producción significativo; 2) se trata de un tema de interés que se encuentra en auge, demostrando la ley de Prince; 3 ) con el cumplimiento de la ley de Lotka y la ley de Bradford se confirma que la mayor parte de la producción se concentra en un número reducido de autores y revistas, demostrando que existe una especialización de esta temática por parte de autores concretos que publican frecuentemente sobre esta temática, destacando al autor Alloway y Gathercole; 4)también se demuestra la especialización de determinadas revistas que recogen una gran cantidad de artículos que siguen esta misma línea. Destacando la revista Journal Of Research In Special Educational Needs, la revista Dyslexia y Journal Of Learning Disabilities; 5) se trata de un tema que atañe a varias disciplinas del conocimiento, destacando el área de las ciencias sociales y la psicología. Ambas áreas se encuentran entrelazadas y unidas por aspectos referentes a la educación y su mejora desde la equidad. Atendiendo al mapa bibliométrico, se ha podido visualizar la relación que existe entre los distintos conceptos clave que rodean a las competencias lingüísticas, como los factores educativos y psicológicos, las dificultades de aprendizaje y la inclusión educativa; 6 ) en lo que respecta al tipo de documento, se ha comprobado que destaca el artículo de investigación con un $72,94 \%$ de la producción analizada; 7 ) los países donde más documentos se publican sobre este tema, con un $50.97 \%$ del total los documentos, son Estados Unidos y Reino Unido. Por este motivo no resulta extraño que las instituciones más destacadas, a las que están afiliados los autores, pertenezcan a estos mismos países y que sea el inglés el idioma preferente abarcando el $96,12 \%$ de toda la producción literaria que se ha analizado. Son en estas universidades e institutos de investigación donde más interés se muestra por mejorar las competencias lingüísticas fundamentales para la educación, atendiendo a las dificultades de aprendizaje y la diversidad.

\section{AGRADECIMIENTOS}

Estudio financiado a través del Ministerio de Educación, Cultura y Deporte de España a cargo del proyecto de investigación: "Análisis de la animación a la lectura en el alumnado de Educación Primaria en contextos de atención preferente de la zona norte de la ciudad de Granada: las TIC como herramientas incentivadoras "(ref. FPU18/00676)

\section{REFERENCIAS}

Álvarez, C. y Pascual, J., Estudio de caso sobre la formación de lectores críticos mediante textos literarios en la educación primaria, Ocnos, 10, 27-53 (2013)

Aznar, I., Romero, J.M., Rodríguez-García, A.M. y Rodríguez, C., Descubriendo el entorno desde nuevos enfoques: la realidad aumentada como tecnología emergente en educación, En J. Ruiz-Palmero, E. Sánchez-Rivas, y J. SánchezRodríguez (Edit.), Innovación pedagógica sostenible. Málaga: UMA Editorial (2018)

Cain, K., Reading comprehension development and difficulties: an overview. Perspectives on Language and Literacy, 42(2), 9-16 (2016).

Calet, N., Gutiérrez-Palma, N. y Defior, S., Effects of fluency training on reading competence in primary children: the role of prosody, https://doi.org/10.1016/j.learninstruc.2017.04.006, Learning \& Instruction, 52, 59-68 (2017)

Campos Soto, M.N., Ramos-Navas-Parejo, M. y Moreno Guerrero, A.J. Realidad virtual y motivación en el contexto educativo: estudio bibliométrico de los últimos veinte años de Scopus, https://doi.org/10.17163/alt.v15n1.2020.04, ALTERIDAD. Revista de Educación, 15(1), 47-60 (2020)

Clarke, P.J., Snowling, M.J., Truelove, E., y Hulme, C., Ameliorating children's reading-comprehension difficulties: a randomized controlled trial, https://doi.org/10.1177/0956797610375449, Psychological Science, 21(8), 1106-1116 (2010)

Defior, S., Gutiérrez-Palma N. y Serrano, F., Dificultades específicas de aprendizaje, Síntesis, Madrid, España (2015)

Gallego, J.L. y Rodríguez, A., Líneas de investigación sobre educación especial en España: un estudio bibliométrico (2006-2010), Revista de Ciencias Sociales, 21(2), 219-233 (2015) 
González-Gil, F., Martín-Pastor, E., Poy, R. y Jenaro, C., Percepciones del profesorado sobre la inclusión: estudio preliminar, http://dx.doi.org/10.6018/reifop.19.3.219321, Revista Electrónica Interuniversitaria de Formación del Profesorado, 19(3), 11-24 (2016)

Hong, H.Y., Ma, L., Lin, P.Y., Yuan-Hsuan Lee, K., Advancing third graders' reading comprehension through collaborative knowledge building: a comparative study in Taiwan, https://doi.org/10.1016/j.compedu.2020.103962, Computers and Education, 157,103962 (2020)

Igwesi, U., Chimah, J.N., y Nwachukwu, V.N. The use of ICTs and audiovisual resources in developing children's reading habits in Nigeria. Library Philosophy and Practice, (1), 825 (2012)

Instituto Nacional de Evaluación Educativa, PIRLS, http://www.educacionyfp.gob.es/inee/evaluacionesinternacionales/pirls/pirls-2016.html, (2016)

Jerrim, J., y Moss, G., The link between fiction and teenagers' reading skills: International evidence from the OECD PISA study, https://doi.org/10.1002/berj.3498, British Educational Research Journal, 45(1), 181-200 (2019)

Jiménez-García, M., y Martínez-Ortega, M.Á. (2017). El uso de una aplicación móvil en la enseñanza de la lectura, https://dx.doi.org/10.4067/S0718-07642017000100015, Información Tecnológica, 28(1), 151-160 (2017)

Karlidag-Dennis, E., Hazenberg, R., y Dinh, A.T., Is education for all? the experiences of ethnic minority students and teachers in north-western Vietnam engaging with social entrepreneurship, https://doi.org/10.1016/j.ijedudev.2020.102224, International Journal of Educational Development, 77,102224 (2020)

Lai, S.A., George Benjamin, R., Schwanenflugel, P.J., y Kuhn, M.R., The longitudinal relationship between reading fluency and reading comprehension skills in second-grade children. https://doi.org/10.1080/10573569.2013.789785, Reading \& Writing Quarterly, 30(2), 116-138 (2014)

Li, X., Mok, S.W., Cheng, Y.Y.J., y Chu, S.K.W., An examination of a gamified e-quiz system in fostering students' reading habit, interest and ability, https://doi.org/10.1002/pra2.2018.14505501032, Proceedings of the Association for Information Science and Technology, 55(1), 290-299 (2018)

Miranda, A., Bibliometría. Bibliotecas, 8(1), 1-11 (1990)

Neumann, H., Leu, S., McDonough, K. y Crawford, B., Improving students' source integration skills: does a focus on reading comprehension and vocabulary development work?, https://doi.org/10.1016/j.jeap.2020.100909, Journal of English for Academic Purposes, 48,100909 (2020)

Prasanna-Kumara, B.M., Evaluation of ICT impact on reading habits of students in Amrita Vishwa Vidyapeetham Bengaluru, Library Philosophy and Practice, 2973, 1-7 (2019).

Price, D.J.S., Little Science, big science.and beyond. Nueva York, NY, Columbia University Press (1986).

Retelsdorf, J., y Köller, O., Reciprocal effects between reading comprehension and spelling. https://doi.org/10.1016/j.lindif.2013.11.007, Learning and Individual Differences, 30, 77-83 (2014)

Ripoll, J., y Aguado, G., La mejora de la comprensión lectora en español: un meta-análisis, https://doi.org/10.1387/RevPsicodidact.9001, Revista de Psicodidáctica, 19, 27-44 (2014)

Rodríguez-Fuentes, A., Evaluación de dificultades lectoras escolares, En Entornos y estrategias educativas para la inclusión social, Comares, Granada, España (2017)

Rodríguez, A., y Gallego, J.L., Análisis bibliométrico sobre educación especial. Profesorado, https://doi.org/10.30827/profesorado.v23i1.52458, Revista de Currículum y Formación del Profesorado, 23(1), 307-327 (2019)

Secul-Giusti, C., y Viñas, M., Fomento de la lectura a través de las redes sociales, Letras, 2, 29-36 (2015)

Serna, M., Rodríguez, A., y Etxaniz, X., Biblioteca escolar y hábitos lectores en los escolares de educación primaria, http://dx.doi.org/10.18239/ocnos_2017.16.1.1205, Ocnos, 16(1), 18-49 (2017)

Taghinezhad, A., y Riasati, M.J., The interplay of critical thinking explicit instruction, academic writing performance, critical thinking ability, and critical thinking dispositions: an experimental study, IJERI: International Journal of Educational Research and Innovation, (13), 143-165 (2020)

Triviño, L.S., Sola, T. y Rivas, M.A., Comprensión lectora y gráficos estadísticos en alumnos de cuarto grado de primaria, Educere, 17(58), 455-464 (2013)

Valdés, M., ¿Leen en forma voluntaria y recreativa los niños que logran un buen nivel de comprensión lectora?, https://doi.org/10.18239/ocnos_2013.10.04, Ocnos, 10, 71-89 (2013) 